\title{
NÚMERO DE ESPECIES EN FUNCIÓN DEL DIÁMETRO MÍNIMO EVALUADO EN BOSQUES MONTANOS Y PREMONTANOS DE LA SELVA CENTRAL DEL PERÚ
}

\section{NUMBER OF SPECIES ACCORDING TO MINIMUM DIAMETER MEASURED IN THE MONTANE AND PREMONTANE FORESTSFROM SELVA CENTRAL OF PERU}

\author{
Carlos Reynel ${ }^{1,2}$, Robin Fernandez-Hilario ${ }^{1}$, Flavio Quintero ${ }^{1}$, Belvi Cáceres ${ }^{3}$ y \\ Sonia Palacios-Ramos ${ }^{1}$
}

\begin{abstract}
Resumen
Se emplean datos de ocho Parcelas Permanentes de 1 ha (diámetro mínimo de evalua ción $10 \mathrm{~cm}$ de DAP) y un número similar de Transectos RAP de 0.1 ha (diámetro mínimo de evaluación $2.5 \mathrm{~cm}$ de DAP) emplazados en bosques húmedos premontanos $(940-1150 \mathrm{msnm})$ y montanos primarios (2 078 - $2770 \mathrm{msnm}$ ) del valle de Chanchamayo (Departamento de Junín / Perú), con el objetivo de mostrar la influencia del diámetro mínimo de registro en la diversidad arbórea hallada. Se ana lizan los porcentajes de especies por clase diamétrica y los correspondientes valores de Alpha de Fisher. Los resultados muestran que inventarios considerando solamente diámetros por encima de $40 \mathrm{~cm}$ de DAP capturan muy poco de la riqueza existente en el bosque (equivalente a sólo el $29.2 \%$ y $22.9 \%$ del totalde las especies en bosques premontanos y montanos, respectivamente); los valores de Alpha de Fisher son coincidentes con lo mencionado.
\end{abstract}

Palabras clave: Chanchamayo, diversidad arbórea, Parcelas Permanentes, sistema de cla sificación APG, Transectos RAP.

\begin{abstract}
Data from eight Permanent Plots of 1 ha (including all plants $\geq 10 \mathrm{~cm}$ ) and similar number of RAP Transects of 0.1 ha (including all plants $\geq 2.5 \mathrm{~cm}$ ) established in primary tropical humid forests, premontane (940 - $1150 \mathrm{masl})$ and montane (2 078 - $2770 \mathrm{masl})$ in the Chanchamayo valley (Junin / Peru) are here used to finding to which extent the minimum recorded diameter influences tree diversity as found in forest inventories. In each diameter class, both the species percents and Fisher's Alpha values are considered. Our results show that forest inventories including only large diameters, e.g. $\geq 40 \mathrm{~cm} \mathrm{DBH}$, capture a low percent of the species $(29.2 \%$ and $22.9 \%$ in premontane and montane forests, respectively); Fisher's Alpha values are coincident with these findings.

Key words: Chanchamayo, tree diversity, Permanent Plots, APG classification system, RAP Transects.
\end{abstract}

\section{Introducción}

En el Perú, una antigua corriente de evaluaciones del componente arbóreo de los bosques, comprendida bajo el término "inventario forestal", desarrolladas mayormente entre las décadas 1970 - 2000, estuvo enfocada en reflejar, de manera central, los volúmenes maderables presentes en áreas boscosas, considerando sobre todo los árboles de maderas valiosas y diámetros mayores, por ejemplo, a quellos superiores a 35 o $40 \mathrm{~cm}$ de Diámetro a la Altura del Pecho (DAP) (Malleux, 1982; MINAG, 2012). Estos inventarios obviaron la identificación científica verificable de las especies, encarándola mayormente con una metodología de reconocimiento de árboles en el ca mpo bajo sus nombres comunes por personal local no capacitado en taxonomía, y sin el respaldo de colecciones botánicas e identificaciones científicas comprobables (Vásquez \& Gentry, 1987).

Con el paso del tiempo, se ha hecho evidente el valor significativo de la biodiversidad, así como el de la diversidad de productos forestales no maderables, y también el hecho de que éstos juegan un papel fundamental en los ciclos ecológicos de los cuales depende la supervivencia del hombre y otros orga nismos (Dinerstein et al., 2020). Por estas razones, las evaluaciones actuales de la vegetación arbórea incluyen cada vez más, a las especies de árboles que tienen tamaños menores, pero pueden ser importantes en la perspectiva mencionada.

En este contexto debe considerarse la extra ordinaria diversidad biológica albergada en territorio peruano. Los notables niveles de diversidad de árboles existentes en los bosques del país no fueron revelados en detalle hasta estudios conducidos a partir de la década de los 80s, cuando evaluaciones desarrolladas en Parcelas Permanentes (PP), con metodologías de identificación taxonómica verificable, evidenciaron que en espacios como la llanura amazónica peruana, la diversidad arbórea (Diversidad Alfa o número de especies por unidad de área) puede alcanzar récords de hasta 300 


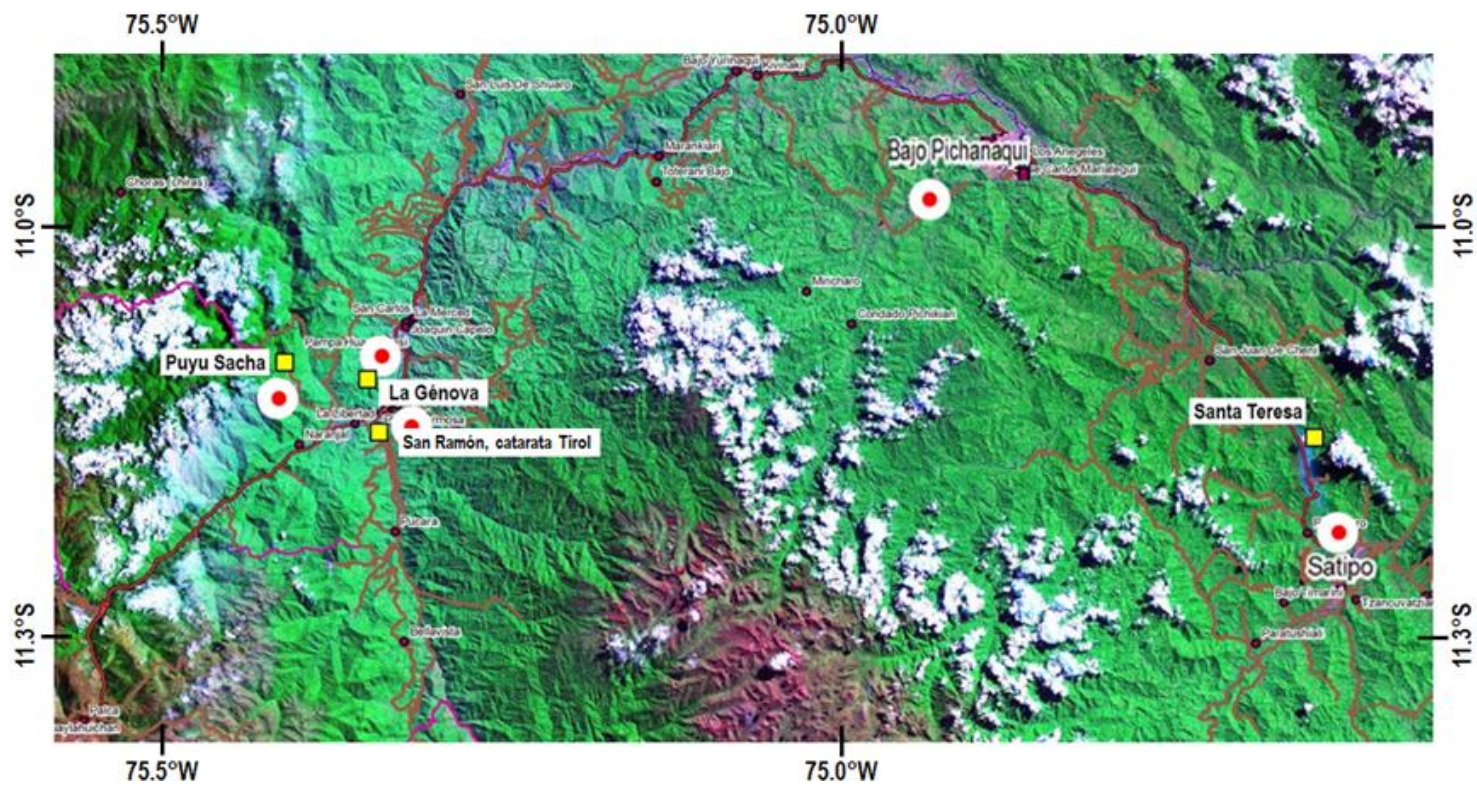

Figura 1. Mapa del ámbito de estudio, los cuadrados a marillos indican la ubicación de las tres estaciones de investigación y la catarata de Tirol donde se instalaron las Parcelas Permanentes y Transectos RAP, en el Departamento de Junín / Perú.

especies por hectárea (Gentry, 1988, 1989, 1995; Ter Steege et al., 2003).

Las primeras cuantificaciones científicamente sustentadas de la cantidad total de especies de árboles existentes en el Perú evidenciaron, desde inicios de los 90s, que el país posee vastos espacios en los cuales las especies de árboles existentes son desconocidas (Honorio \& Reynel, 2003). Estimaciones actuales sugieren que el $20 \%$ - 30\% de la flora arbórea peruana no está descrita, cataloga da ni conocida por la ciencia; particularmente, existen áreas que son auténticos vacíos de conocimiento en tal sentido (Honorio \& Reynel, 2003; Joppa et al., 2011).

La necesidad actual, de evaluar la biodiversidad de los bosques, reconoce la importancia del estrato arbóreo como templete sobre el cual conjuntos de otros organismos encuentran sus nichos habitables; por ello, la cuantificación de esta diversidad es empleada actualmente como una herramienta para conocer el valor y estado de conservación de las áreas forestales (Antón \& Reynel, 2004).

Los levantamientos de unidades muestrales para el estudio de áreas de bosque en el Neotrópico, y de modo particular en el Perú, establecidas con metodologías apropiadas para la identificación de las especies de flora, han ido en aumento en décadas recientes (Antón \& Reynel, 2004; Marcelo \& Reynel, 2014; De Rutte \& Reynel, 2016). En ese contexto, es relevante la actividad del consorcio interinstitucional RAINFOR, que lidera el levantamiento de PP a nivelde los trópicos de todo el mundo, con énfasis en el Neotrópico (RAINFOR, 2000). No obstante, uno de los aspectos que no ha sido documentado para las formaciones boscosas del país es la manera como el diámetro mínimo, considerado en una evaluación, podría influir en la diversidad de especies hallada.

En un estudio conducido en un bosque húmedo tropical de tierra firme en la Guayana Francesa (Bordenave, 1998), de un total de 203 especies de plantas vasculares $\geq 2.5 \mathrm{~cm}$ DAP registradas, $40 \%$ de las especies tuvieron $\geq 2.5 \mathrm{~cm}$ de DAP; $21 \%$ tuvieron $\geq$ $10 \mathrm{~cm}$ DAP y solamente $8 \%$ tuvieron $\geq 25 \mathrm{~cm}$ DAP. Una investiga ción llevada a cabo en un bosque húmedo tropical semideciduo en Uganda, África, para un total de 32 parcelas de 0.5 ha, se tuvo un registro totalde 269 especies de plantas vasculares $\geq 2 \mathrm{~cm}$ DAP, y la cantidad de especies $\geq 10 \mathrm{~cm}$ DAP disminuyó a 216 (Mwavu \& Witkowski, 2015). Estos estudios muestran que cuando el diámetro limitante en la evaluación es mayor, el número de especies disminuye considerablemente.

El objetivo del presente trabajo fue mostrar la relación existente entre los diámetros mínimos considerados en una evaluación de la diversidad arbórea y la cantidad de especies que dicha evaluación podría registrar. Para ello, se analizaron los datos de áreas de bosque en dos diferentes formaciones ecológicas en la selva central peruana: los bosques húmedos montanos nublados y los bosques húmedos premontanos. La hipótesis es que cuando el diámetro limitante de la evaluación es mayor, el número de especies y familias disminuye considerablemente.

\section{Materiales y métodos}

Área de estudio; sus características ecológicas y de población humana

El ámbito de estudio comprendió bosques emplazados a cierta distancia del eje carretero que 
Tabla 1. Ubicación de las Parcelas Permanentes de 1 ha usadas en bosques húmedos del e strato premontano y montano en el Departamento de Junín / Perú.

\begin{tabular}{|c|c|c|c|c|c|c|c|c|}
\hline \multirow{2}{*}{ Núm. } & \multirow{2}{*}{$\begin{array}{c}\text { Código } \\
\text { de } \\
\text { Parcela }\end{array}$} & \multirow{2}{*}{$\begin{array}{l}\text { Localización } \\
(*)\end{array}$} & \multirow{2}{*}{ Provincia } & \multicolumn{3}{|c|}{ Coordenadas UTM } & \multirow{2}{*}{$\begin{array}{l}\text { Elevación } \\
\text { (msnm) }\end{array}$} & \multirow{2}{*}{$\begin{array}{c}\text { Estrato } \\
\text { elevacional }\end{array}$} \\
\hline & & & & Zona & Este & Sur & & \\
\hline 01 & P-GC & LG & Chanchamayo & $18 \mathrm{~L}$ & 461821 & 8772580 & 1150 & Premontano \\
\hline 02 & P-GL & LG & Chanchamayo & $18 \mathrm{~L}$ & 461983 & 8772505 & 1075 & Premontano \\
\hline 03 & P-SRL & SR & Chanchamayo & $18 \mathrm{~L}$ & 464750 & 8769200 & 1150 & Premontano \\
\hline 04 & P-SPI & ST & Satipo & $18 \mathrm{~L}$ & 538044 & 8765986 & 940 & Premontano \\
\hline 05 & P-PL & APRODES & Chanchamayo & $18 \mathrm{~L}$ & 453050 & 8773950 & 2100 & Montano \\
\hline 06 & P-PL2 & APRODES & Chanchamayo & $18 \mathrm{~L}$ & 453373 & 8773935 & 2078 & Montano \\
\hline 07 & P-PR & APRODES & Chanchamayo & $18 \mathrm{~L}$ & 452425 & 8774515 & 2275 & Montano \\
\hline 08 & P-PA & APRODES & Chanchamayo & $18 \mathrm{~L}$ & 451870 & 8772223 & 2770 & Montano \\
\hline
\end{tabular}

(*) Localizaciones: LG = Estación Fundo La Génova UNALM; SR = San Ramón, catarata de Tirol; ST = Estación Fundo Santa Teresa

UNALM; APRODES = Estación del bosque Puyu Sacha de la Asociación Peruana para la Promoción del Des arrollo Sostenible.

discurre desde Lima / Perú hacia el Este, en la gradiente elevacional del flanco oriental andino, con acceso desde las ciudades de Tarma, San Ramón, La Merced y Satipo. Políticamente, corresponde a las provincias de Chanchamayo y Satipo del Departamento de Junín / Perú. Este espacio, que histórica mente ha constituido el paisaje forestal más prontamente colonizado desde la capital, Lima, se encuentra actualmente muy alterado por la presencia humana, sobre todo en las partes más accesibles, de pendiente suave o moderada. En las zonas con menos accesibilidad, existen remanentes de bosques más o menos extensos, incluyendo extensiones que han sido priorizadas para la conservación. Las actividades económicas más importantes para la población asentada son la producción de frutas tropicales, para las cuales los valles allí existentes constituyen una despensa frutícola primaria para la capital; la producción de café y, también, el turismo local (INEI, 1993).

Las localizaciones estudiadas corresponden a bosques húmedos montanos nublados, en el tercio alto de la cuenca del río Perené, entre 2000 - 2800 msnm, y progresivamente hacia el Este, bosques húmedos premontanos situados entre $600-2000 \mathrm{msnm}$. De acuerdo con el Sistema de Clasificación Ecológica por Zonas de Vida de Holdridge (Holdridge, 1978; INRENA, 1995), están ubicadas principalmente en Bosque húmedo Premontano Tropical y Bosque muy húmedo Premontano Tropical. Las localizaciones de estudio tienen acceso desde las ciudades de San Ramón y La Merced, en el valle de Chanchamayo, y Satipo, en el tercio medio del río Perené. El trabajo ha estado centrado en tres estaciones de investigación: Puyu Sacha (APRODES), Fundo Génova y Fundo Santa Teresa; las dos última sertenecen alInstituto Regional de Desarrollo (IRD-Selva) de la UNALM (Figura 1).

Unidades de muestra consideradas

OCHO PARCELAS PERMANENTES (PP) DE 1 HA.

Establecidas previamente (Antón \& Reynel, 2004; Caro et al., 2004; Reynel \& Honorio, 2004; Marcelo \& Reynel, 2014; De Rutte \& Reynel, 2016), éstas son parcela s mayormente de forma cuadra da, de $100 \times 100$ $\mathrm{m}$; en pocos casos rectangulares (Tabla 1). Están subdivididas en 25 subparcelas de $20 \times 20 \mathrm{~m}$ empleando la metodología del Consorcio de Investigación RAINFOR (Phillips et al., 2009), y son mantenidas para estudios vinculados a la dinámica de los bosques. En cada PP se han codificado y evaluado todos los árboles $\geq 10 \mathrm{~cm}$ de DAP. Para cada árbol, fue registrado el DAP y la altura total, y fue colectada una muestra botánica, que preparada y acondicionada de acuerdo a la metodología estándar(Bridson \& Forman, 1999; Maden, 2004), fue deposita da en el Herbario de la Facultad de Ciencias Forestales de la Universidad Nacional Agraria - La Molina, Lima, donde fue determinada por los dos primeros autores y botánicos especialistas que han revisado las colecciones del herbario. Se ha empleado el sistema de clasificación taxonómico por familias del Angiosperm Phylogeny Group (APG, 2016).

OCHO TRANSECTOS RAP DE $0.1 \mathrm{HA}$.

Estos fueron transectos de $2 \times 500 \mathrm{~m}$, subdivididos en tramos de $50 \mathrm{~m}$; los posicionamientos de sus puntos iniciales fueron registrados, así como su orientación geográfica; más, los transectos, no son unidades mantenidas permanentemente en el tiempo. En cada Transecto RAP fueron registradas todas las plantas $\geq$ $2.5 \mathrm{~cm}$ de DAP; cada planta fue numerada de manera sucesiva, consignando el tramo del Transecto RAP al que pertenecía (Phillips \& Miller, 2002; Phillips et al., 2003). Al igual que para las PP, fue colectada para cada una de ellas una muestra botánica, que fue preparada, a condiciona da, deposita da y determina da en el herbario de modo similar. Todos los Transectos RAP corresponden al estra to de bosque húmedo premontano y sus características se muestran en la Tabla 2.

\section{Análisis efectuados}

NÚMERO DE ESPECIES (DIVERSIDAD ALFA)

Se ha calculado la Diversidad Alfa, en este caso expresada como el número de especies por hectárea. Se establecieron clases diamétricas cada $5 \mathrm{~cm}$ para analizar el número de especies (Diversidad Alfa). Se analizaron las PP por separado y, adicionalmente, el 
conjunto de éstas por estrato (bosque premontano y bosque montano) (Tabla 3 ).

Tabla 2. Ubicación de los Transectos RAP de 0.1 ha establecidos en bosques húmedos del estrato premontano en la Estación Fundo La Génova UNALM en la Provincia de Chanchamayo / Departamento de Junín / Perú.

\begin{tabular}{llccr}
\hline \multicolumn{3}{c}{$\begin{array}{c}\text { Código } \\
\text { Transecto }\end{array}$} & \multicolumn{3}{c}{ Coordenadas } & Elevación \\
\cline { 2 - 4 } (msnm)
\end{tabular}

Tabla 3. Clases diamétricas establecidas para el análisis de la Diversidad Alfa en las Parcelas Permanentes y Transectos RAP del presente estudio.

\begin{tabular}{ccc}
\hline \multirow{2}{*}{$\begin{array}{c}\text { Clase } \\
\text { diamétrica }\end{array}$} & \multicolumn{2}{c}{ Intervalos (cm) } \\
\cline { 2 - 3 } & $\begin{array}{c}\text { Parcelas } \\
\text { Permanentes }\end{array}$ & $\begin{array}{c}\text { Transectos } \\
\text { RAP }\end{array}$ \\
\hline 1 & $10-14.99$ & $2.5-4.99$ \\
2 & $15-19.99$ & $5-9.99$ \\
3 & $20-24.99$ & $10-14.99$ \\
4 & $25-29.99$ & $15-19.99$ \\
5 & $30-34.99$ & $20-24.99$ \\
6 & $35-39.99$ & $25-29.99$ \\
7 & $>40$ & $30-34.99$ \\
8 & - & $35-39.99$ \\
9 & - & $>40$ \\
\hline
\end{tabular}

\section{CURVAS DE ACUMULACIÓN DE ESPECIES}

Usando el programa EstimateS 9.1 (Colwell, 2013), se elaboraron curvas de acumulación de especies basadas en la Diversidad Alfa registrada para cada PP y Transecto RAP en cada estrato elevacional (premontano y montano). Adicionalmente, con el mismo programa se evaluó el desempeño de las curvas correspondientes al número de especies representadas por un individuo ("singletons") y al número de especies representadas solamente en una subparcela ("uniques"). Se hizo esto para poder contrastarlas con la diversidad promediada (S Mean) y analizar cuán completo fue el inventario obtenido. Existe cierto consenso sobre que las curvas de "singletons" y "uniques" descendentes o a sintóticas indicarían que el muestreo florístico realizado ha sido eficaz (Villareal et al., 2004; Cava et al., 2015).

\section{ALPHA FISHER}

Se calculó el índice de Diversidad Alpha Fisher para las PP mediante el programa PAST versión 1.91 (Hammer et al., 2001). Estos cálculos se realizaron para todas las especies e individuos mayores a 10,20,30 y $40 \mathrm{~cm}$ de DAP en el estrato premontano y montano.

\section{Resultados \\ Diversidad Alfa por clase diamétrica}

PARCELAS PERMANENTES - DIVERSIDAD ALFA POR CLASE DIAMÉTRICAEN EL ESTRATO PREMONTANO

En las Tabla 4 se muestran los resultados de números de individuos y especies hallados en PP en bosques premontanos (elevaciones entre 600 - 1800 msnm). En todos los casos, se trata de bosques húmedos maduros, y sin intervención humana.

La observación de los datos de las PP en el estrato premontano muestra que, en todos los casos, la primera clase diamétrica, $10-14.99 \mathrm{~cm}$ de diámetro, registra porcentajes más altos del totalde individuos (promedio $=36.8 \%$ ), y del totalde especies (promedio $=63.1 \%$ ) . Dichos porcentajes corresponden, en todos los casos, a más de la mitad y hasta más de dos tercios del totalde la Diversidad Alfa hallada. La siguiente clase diamétrica, 15 - $19.99 \mathrm{~cm}$, también contiene un porcentaje elevado del total de individuos (promedio $=$ $24.1 \%$ ) y del totalde especies (promedio $=48.87 \%$ ). En contraste, en las clases dia métricas mayores, a partir de $40 \mathrm{~cm}$, se observa una disminución drástica delnúmero de individuos y su porcentaje en relación al total (promedio $=7.1 \%), \mathrm{y}$ de especies registradas $\mathrm{y} \mathrm{su}$ porcentaje en relación al total (promedio $=22.9 \%$ ) (Figura 2).

PARCELAS PERMANENTES - DIVERSIDAD ALFA EN EL ESTRATO MONTANO

En la Tabla 5 se muestran los resultados del número de individuos y especies halladas en PP de bosques montanos (elevaciones entre $1800-2800 \mathrm{msnm}$ ). En todos los casos, se trata de bosques húmedos maduros, y sin intervención humana.

Tabla 4. Número (y porcentaje) de individuos y especies por clase diamétrica en las Parcelas Permanentes evaluadas en el estrato premontano de la Selva Centraldel Perú.

\begin{tabular}{|c|c|c|c|c|c|c|c|c|}
\hline \multirow[b]{2}{*}{$\begin{array}{l}\text { Clase } \\
\text { Diam. }\end{array}$} & \multicolumn{2}{|c|}{ P-GC } & \multicolumn{2}{|c|}{ P-GL } & \multicolumn{2}{|c|}{ P-SRL } & \multicolumn{2}{|c|}{ P-SPI } \\
\hline & $\begin{array}{c}\text { Núm. } \\
\text { inds. }(\%)\end{array}$ & $\begin{array}{c}\text { Núm. spp. } \\
(\%)\end{array}$ & $\begin{array}{c}\text { Núm. } \\
\text { inds. }(\%)\end{array}$ & $\begin{array}{c}\text { Núm.spp. } \\
(\%)\end{array}$ & $\begin{array}{c}\text { Núm. } \\
\text { inds. }(\%)\end{array}$ & $\begin{array}{c}\text { Núm. spp. } \\
(\%)\end{array}$ & $\begin{array}{c}\text { Núm. } \\
\text { inds. }(\%)\end{array}$ & $\begin{array}{c}\text { Núm. spp. } \\
(\%)\end{array}$ \\
\hline 1 & 189 (37.6) & $71(65.1)$ & $165(38.7)$ & $49(75.4)$ & $180(39.1)$ & $70(56.9)$ & $245(31.9)$ & $94(55.0)$ \\
\hline 2 & $132(26.2)$ & $62(56.9)$ & $115(27.0)$ & $33(50.8)$ & $96(20.9)$ & $54(43.9)$ & $172(22.4)$ & 75 (43.9) \\
\hline 3 & 57 (11.3) & $33(30.3)$ & 57 (13.4) & $25(38.5)$ & 77 (16.7) & 38 (30.9) & 145 (18.9) & 69 (40.4) \\
\hline 4 & $49(9.7)$ & $31(28.4)$ & $22(5.2)$ & $15(23.1)$ & $37(8.0)$ & $21(17.1)$ & $74(9.6)$ & $45(26.3)$ \\
\hline 5 & $29(5.8)$ & $23(21.1)$ & $15(3.5)$ & $12(18.5)$ & $22(4.8)$ & $16(13.0)$ & $60(7.8)$ & $36(21.1)$ \\
\hline 6 & $16(3.2)$ & $12(11.0)$ & $11(2.6)$ & $10(15.4)$ & $13(2.8)$ & $7(5.7)$ & $33(4.3)$ & $26(15.2)$ \\
\hline 7 & $31(6.2)$ & $21(19.3)$ & $41(9.6)$ & 29 (44.6) & $35(7.6)$ & $17(13.8)$ & $38(5.0)$ & 24 (14.0) \\
\hline
\end{tabular}


Tabla 5. Número (y porcentaje) de individuos y especies por clase diamétrica en las Parcelas Permanentes evaluadas en el estrato montano de la Selva centraldel Perú.

\begin{tabular}{|c|c|c|c|c|c|c|c|c|}
\hline \multirow{2}{*}{$\begin{array}{l}\text { Clase } \\
\text { Diam }\end{array}$} & \multicolumn{2}{|c|}{ P-PL } & \multicolumn{2}{|c|}{ P-PL2 } & \multicolumn{2}{|c|}{ P-PR } & \multicolumn{2}{|c|}{ P-PA } \\
\hline & $\begin{array}{c}\text { Núm. } \\
\text { inds. }(\%)\end{array}$ & $\begin{array}{c}\text { Núm. spp. } \\
(\%)\end{array}$ & $\begin{array}{c}\text { Núm. } \\
\text { inds. }(\%)\end{array}$ & $\begin{array}{c}\text { Núm.spp. } \\
(\%)\end{array}$ & $\begin{array}{c}\text { Núm. } \\
\text { inds. }(\%)\end{array}$ & $\begin{array}{c}\text { Núm.spp. } \\
(\%)\end{array}$ & $\begin{array}{c}\text { Núm. } \\
\text { inds. }(\%)\end{array}$ & $\begin{array}{c}\text { Núm. spp. } \\
(\%)\end{array}$ \\
\hline 1 & $252(34.3)$ & $98(62.8)$ & $249(35.9)$ & $85(70.8)$ & $277(48.2)$ & $51(68.0)$ & $170(35.9)$ & $33(73.3)$ \\
\hline 2 & 154 (21) & 68 (43.6) & $150(21.6)$ & $63(52.5)$ & $118(20.5)$ & $45(60.0)$ & 104 (22) & $27(60.0)$ \\
\hline 3 & $105(14.3)$ & 49 (31.4) & 88 (12.7) & $43(35.8)$ & $58(10.1)$ & $29(38.7)$ & $62(13.1)$ & $19(42.2)$ \\
\hline 4 & $70(9.5)$ & $38(24.4)$ & $68(9.8)$ & $33(27.5)$ & $37(6.4)$ & $22(29.3)$ & $54(11.4)$ & 20 (44.4) \\
\hline 5 & $44(6)$ & $28(17.9)$ & $56(8.1)$ & $33(27.5)$ & $35(6.1)$ & $21(28.0)$ & $33(7)$ & $11(24.4)$ \\
\hline 6 & $36(4.9)$ & $27(17.3)$ & $37(5.3)$ & $22(18.3)$ & $17(3)$ & $13(17.3)$ & $18(3.8)$ & $8(17.8)$ \\
\hline 7 & 73 (9.9) & $39(25)$ & $45(6.5)$ & $25(20.8)$ & $33(5.7)$ & $18(24)$ & $32(6.8)$ & $10(22.2)$ \\
\hline
\end{tabular}

En cuanto al estrato montano, los datos de las PP muestran, de manera similar, que en todos los casos la primera clase diamétrica $(10-14.99 \mathrm{~cm}$ de diámetro $)$ registra porcentajes más altos del total de individuos (promedio $=38.5 \%$ ), y del totalde especies (promedio $=68.8 \%$ ). Esos porcentajes corresponden, en todos los casos, desde más de la mitad hasta dos tercios del total de la Diversidad Alfa hallada. La siguiente clase diamétrica $(15-19.99 \mathrm{~cm})$ también contiene un porcentaje elevado del total de individuos (promedio $=$ $21.27 \%$ ), y del total de especies (promedio $=54.0 \%$ ). En contraste, en las cla ses diamétricas mayores (a partir de $40 \mathrm{~cm}$ ) se observa una disminución drástica del número de individuos y su porcentaje en relación al total (promedio $=7.2 \%$ ), y de especies registradas y su porcentaje en relación al total (promedio $=23 \%$ ) (Figura 3). Los datos reflejan, entonces, que una evaluación que incluya solo árboles de diámetros mayores a $40 \mathrm{~cm}$, al igual que en los bosques premontanos, tendría una resolución muy baja del componente de Diversidad Alfa en el espacio estudiado.

SÍNTESIS DE PARCELAS PERMANENTES - NÚMERO

DE ESPECIES Y FAMILIAS DE ACUERDO AL DIÁMETRO MÍNIMO DE EVALUACIÓN EN AMBOS ESTRATOS (PREMONTANO Y MONTANO)

Los datos hallados muestran que cuando las evaluaciones del componente arbóreo del bosque son realizadas con DAP mínimos de evaluación progresivamente mayores, la Diversidad Alfa registrada disminuye considerablemente. Esto se puede observaren las Tablas 6 y 7. En el estrato premontano, si se considera un DAP mínimo de $40 \mathrm{~cm}$, la cantidad de especies registradas disminuye a 13.8 - 44.6\% (promedio $29.2 \%$ ) del total registrado para un DAP mínimo de $10 \mathrm{~cm}$. Para el estrato montano, la Diversidad Alfa registrada disminuye a 20.8 - 25\% (promedio $22.9 \%$ ) del total registrado para un DAP mínimo de $10 \mathrm{~cm}$. Asimismo, el comportamiento del índice Alpha de Fisher es coincidente con lo mencionado. Dicho índice corrige la distorsión en los valores de diversidad origina da por las diferencias en el número de individuos entre los datos (Tabla 6).

\section{Curvas de acumulación}

Hemos elaborado las curvas de acumulación de especies basadas en la Diversidad Alfa registrada para cada PP en cada estrato elevacional (premontano y montano). Para el estrato premontano se pudo observar que las curvas de "singletons" y "uniques" son a sintóticas sólo para las parcelas P-GC y P-GL (ambas en La Génova), lo que indicaría que en las PP restantes en ese estrato el muestreo fue sólo relativamente eficaz y podría no haber capturado el grueso de la Diversidad Alfa estimada. Por otro lado, en las PP del estrato montano las curvas de "singletons" y "uniques" sí presentaron tendencias asintóticas, indicando que las unidades de muestra levantadas habrían capturado de manera bastante completa la Diversidad Alfa allí existente (Figuras 4 y 5 ).

Muchos de los géneros registrados en los bosques premontanos también están presentes en los bosques montanos. Sin embargo, algunos están restringidos a estratos superiores, como los géneros Gordonia (Theaceae), Podocarpus (Podocarpaceae), Symplocos (Symplocaceae) o Weinmannia (Cunoniaceae),

Tabla 6. Número (y porcentaje) de especies y Alpha Fisher en función del diámetro mínimo de evaluación en las Parcelas Permanentes en el estrato premontano y montano de la Selv a Central del Perú.

\begin{tabular}{llrrrl}
\hline \multirow{2}{*}{$\begin{array}{c}\text { Código } \\
\text { de } \\
\text { parcela }\end{array}$} & Estrato & \multicolumn{4}{c}{ Número total de especies (y porcentaje); Alpha Fisher } \\
\cline { 2 - 6 } & & $\mathbf{~ 1 0 ~ c m ~ D A P ~}$ & $>\mathbf{2 0 ~ c m ~ D A P}$ & $>\mathbf{3 0}$ cm DAP & $>$ 40 cm DAP \\
\hline P-GC & Premontano & $109(100) ; 42.83$ & $65(59.6) ; 36.17$ & $45(41.3) ; 46.38$ & $21(19.3) ; 28.6$ \\
P-GL & Premontano & $65(100) ; 21.37$ & $49(75.4) ; 25.88$ & $36(55.4) ; 31.69$ & $29(44.6) ; 44.16$ \\
P-SRL & Premontano & $123(100) ; 54.98$ & $67(54.5) ; 37.92$ & $32(26) ; 22.79$ & $17(13.8) ; 13.03$ \\
P-SPI & Premontano & $171(100) ; 68.29$ & $116(67.8) ; 60.65$ & $63(36.8) ; 47.7$ & $24(14) ; 27.97$ \\
P-PL & Montano & $156(100) ; 60.63$ & $96(61.5) ; 45.67$ & $64(41) ; 41.36$ & $39(25) ; 34.04$ \\
P-PL2 & Montano & $120(100) ; 41.89$ & $74(61.7) ; 31.81$ & $48(40) ; 26.11$ & $25(20.8) ; 23.16$ \\
P-PR & Montano & $75(100) ; 23.03$ & $47(62.7) ; 20.68$ & $33(44) ; 19.81$ & $18(24) ; 16.21$ \\
P-PA & Montano & $45(100) ; 12.22$ & $30(66.7) ; 9.81$ & $19(42.2) ; 7.71$ & $10(22.2) ; 4.99$ \\
\hline
\end{tabular}


Tabla 7. Número (y porcentaje) de familias en función del diámetro mínimo de evaluación en las Parcelas Permanentes en el estrato premontano y montano de la Selva Central del Perú.

\begin{tabular}{llcccc}
\hline $\begin{array}{c}\text { Código } \\
\text { de } \\
\text { Parcela }\end{array}$ & Estrato & > & \multicolumn{3}{c}{ Número total de familias APG (2016) (y porcentaje) } \\
\cline { 2 - 5 } P-GC & Premontano & $41(100)$ & > 20 cm DAP & $>$ 30 cm DAP & $>$ 40 cm DAP \\
P-GL & Premontano & $28(100)$ & $33(80.5)$ & $28(68.3)$ & $14(34.1)$ \\
P-SRL & Premontano & $39(100)$ & $23(82.1)$ & $16(57.1)$ & $14(50)$ \\
P-SPI & Premontano & $47(100)$ & $39(74.4)$ & $18(46.2)$ & $11(28.2)$ \\
P-PL & Montano & $41(100)$ & $36(87.8)$ & $29(61.7)$ & $18(38.3)$ \\
P-PL2 & Montano & $48(100)$ & $39(81.3)$ & $28(68.3)$ & $17(41.5)$ \\
P-PR & Montano & $38(100)$ & $29(76.3)$ & $22(57.9)$ & $18(37.5)$ \\
P-PA & Montano & $22(100)$ & $18(81.8)$ & $13(59.1)$ & $16(42.1)$ \\
\hline
\end{tabular}

característicos de los bosques montanos nublados (Tablas 8 y 9).

Transectos RAP

El conjunto de los ocho Transectos RAP analizados corresponden al estrato premontano. Los resultados del análisis de dichos transectos se muestran en las Tablas 10 y 11. La observación de los datos de los Transectos RAP correspondientes en el estrato premontano (Tabla 10) muestra que, en todos los casos, la primera clase diamétrica $(2.5-4.99 \mathrm{~cm})$ registra los porcentajes más altos del totalde individuos (promedio $=44.6 \%$ ) y del total de especies (promedio $=63.1 \%$ ). Dichos porcentajes corresponden, en todos los casos, a más de la mitad y hasta más de dos tercios del total de la Diversidad Alfa hallada. La siguiente clase diamétrica $(15-19.99 \mathrm{~cm})$ ta mbién contiene un porcentaje elevado del total de individuos (promedio $=28.1 \%$ ) y del total de especies (promedio $=59.7 \%$ ). En contraste, en las clases diamétricas mayores (a partir de $40 \mathrm{~cm}$ ) se observa una disminución drástica del número de individuos y su porcentaje, en relación al total $($ promedio $=1.8 \%), \mathrm{y}$ de especies registradas $\mathrm{y}$ su porcentaje en relación al total (promedio $=8.4 \%$ ) (Figura 6). Empleando este tipo de Transectos, al igual que con PP como unidades de muestra, una evalua ción inclusiva solamente de diámetros mayores a $40 \mathrm{~cm}$ tendría una resolución muy baja del componente de Diversidad Alfa arbórea en el bosque estudiado. Adicionalmente, en la Tabla 11 se pueden apreciarlos géneros con individuos con un diámetro (DAP) mayor a $40 \mathrm{~cm}$.

SÍNTESIS DE TRANSECTOS RAP - NÚMERO DE

ESPECIES Y FAMILIAS DE ACUERDO AL DIÁMETRO MÍNIMO DE EVALUACIÓN EN AMBOS ESTRATOS

Los datos hallados muestran que cuando las evaluaciones del componente arbóreo del bosque son realizadas con DAP mínimos de evaluación progresivamente mayores, la Diversidad Alfa registrada disminuye considerablemente. Esto se puede observar en las Tablas 12 y 13. En el estrato premontano, si se considera un DAP mínimo de $40 \mathrm{~cm}$, la cantidad de especies registradas disminuye a 3.6 $14.3 \%$ (promedio $8.4 \%$ ) del total registrado para un DAP mínimo de $2.5 \mathrm{~cm}$, mientras que, en el caso de las familias, según el sistema de clasificación APG, la cantidad disminuye a 7.7 - 28.6 (promedio 17.4\%).

Tabla 11. Géneros con individuos de un diámetro (DAP) mayor a $40 \mathrm{~cm}$ en los Transectos RAP evaluados en el estrato premontano de la Selva Centraldel Perú.

\begin{tabular}{|c|c|c|c|}
\hline 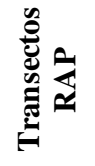 & Géneros & 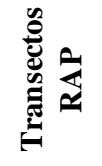 & Géneros \\
\hline $\mathrm{T} 1-30$ & $\begin{array}{l}\text { Cecropia, } \\
\text { Erythrina, } \\
\text { Heliocarpus, } \\
\text { Juglans, Maclura, } \\
\text { Ocotea, Piptadenia }\end{array}$ & T3-40 & $\begin{array}{l}\text { Hieronyma, } \\
\text { Sapium }\end{array}$ \\
\hline $\mathrm{T} 2-30$ & $\begin{array}{l}\text { Cecropia, } \\
\text { Erythrina, Guarea, } \\
\text { Maclura, } \\
\text { Schizolobium }\end{array}$ & $\mathrm{T} 1>50$ & $\begin{array}{l}\text { Ceiba, } \\
\text { Erythrina, } \\
\text { Inga, } \\
\text { Maclura }\end{array}$ \\
\hline $\mathrm{T} 1-40$ & $\begin{array}{l}\text { Erythrina, Ficus, } \\
\text { Inga, Juglans, } \\
\text { Ocotea, } \\
\text { Schizolobium }\end{array}$ & $\mathrm{T} 2>50$ & $\begin{array}{l}\text { Cecropia, } \\
\text { Ficus, Neea, } \\
\text { Ochroma, } \\
\text { Sapium }\end{array}$ \\
\hline $\mathrm{T} 2-40$ & $\begin{array}{l}\text { Acacia, Cecropia, } \\
\text { Erythrina, Inga, } \\
\text { Juglans, Maclura, } \\
\text { Ocotea, Piptadenia, } \\
\text { Pleuranthodendron }\end{array}$ & $\mathrm{T} 3>50$ & $\begin{array}{l}\text { Cecropia, } \\
\text { Ceiba, Ficus }\end{array}$ \\
\hline
\end{tabular}

\section{Discusión}

Es de esperarque el registro de una mayor amplitud de diámetros se traduzca en la presencia de más especies cuando se evalúa la diversidad arbórea en bosques tropicales. Esto se ha evidenciado no sólo en diversos tipos de bosques tropicales. Un estudio realizado en un bosque húmedo semideciduo en Uganda ha mostrado también una cantidad significativamente mayor de especies en diámetros menores (Mwavu \& Witkowski, 2015). En un bosque húmedo de tierra firme, en la Guayana Francesa, se halló bastante menor diversidad de especies en los diámetros por encima de $25 \mathrm{~cm}$ de DAP (19.5\%) (Bordenave et al., 1998). Los datos analizados reafirman que evaluaciones forestales que se restringen 
Tabla 12. Número (y porcentaje) de especies en función del diámetro mínimo de evaluación en los Transectos RAP evaluados (estrato premontano) de la Selva Centraldel Perú.

\begin{tabular}{|c|c|c|c|c|c|}
\hline \multirow{2}{*}{$\begin{array}{l}\text { Código de } \\
\text { Transecto }\end{array}$} & \multicolumn{5}{|c|}{ Número total de especies (Porcentaje) } \\
\hline & $>2.5 \mathrm{~cm} \mathrm{DAP}$ & $>10 \mathrm{~cm} \mathrm{DAP}$ & $>20 \mathrm{~cm} \mathrm{DAP}$ & $>30 \mathrm{~cm}$ DAP & $>40 \mathrm{~cm} \mathrm{DAP}$ \\
\hline T1-30 & $56(100)$ & $33(58.9)$ & $18(32.1)$ & $12(21.4)$ & $7(12.5)$ \\
\hline $\mathrm{T} 2-30$ & $55(100)$ & $35(63.6)$ & $20(36.4)$ & $7(12.7)$ & $5(9.1)$ \\
\hline $\mathrm{T} 1-40$ & $80(100)$ & $37(46.3)$ & $25(31.3)$ & $13(16.3)$ & $7(8.8)$ \\
\hline $\mathrm{T} 2-40$ & $63(100)$ & $37(58.7)$ & $23(36.5)$ & $16(25.4)$ & $9(14.3)$ \\
\hline T3-40 & $56(100)$ & $33(58.9)$ & $12(21.4)$ & $3(5.4)$ & $2(3.6)$ \\
\hline $\mathrm{T} 1>50$ & $63(100)$ & $35(55.6)$ & $22(34.9)$ & $15(23.8)$ & $4(6.3)$ \\
\hline $\mathrm{T} 2>50$ & $75(100)$ & $43(57.3)$ & $24(32)$ & $14(18.7)$ & $6(8)$ \\
\hline $\mathrm{T} 3>50$ & $61(100)$ & $37(60.7)$ & $21(34.4)$ & $13(21.3)$ & $3(4.9)$ \\
\hline
\end{tabular}

solamente a diámetros mayores, por ejemplo (aquellos $\geq 40 \mathrm{~cm}$ de DAP) capturan un porcentaje muy limitado de la diversidad arbórea presente en el bosque (en promedio $23 \%$, tanto para el estrato de bosque premontano como para el estrato montano). Incluso en un estudio comparativo de mayor escala, entre bosques tropicales de Asia, África y América, se encontró que el número de especies de árboles, con diámetros superiores a $30 \mathrm{~cm}$ de DAP, representaba entre el 24 y $32 \%$ de la riqueza (LaFrankie et al., 2006).

Los resultados muestran que la abundancia de individuos se reduce drásticamente en las clases de diámetro superiores. Del total de árboles registrados (individuos por hectárea), aquellos con diámetros iguales o mayores a $40 \mathrm{~cm}$ de DAP representaron entre el 4.9 y $9.6 \%$ para los bosques premontanos y entre el 5.7 y $10 \%$ para bosques montanos. La abundancia en árboles de menor diámetro ha sido reportada en otros estudios del Neotrópico. La notable diferencia entre el número de individuos de las primeras clases diamétricas y las últimas también ha sido destacada en un estudio comparativo entre dos bosques tropicales de Ecuador y Perú. Allí se registró poco más del $40 \%$ de individuos con diámetros de entre 10 y $15 \mathrm{~cm}$, mientras que menos del 3\% alcanzaron diámetros iguales o mayores a $40 \mathrm{~cm}$ de DAP (Pitman et al., 2002). A una mayor escala, cuando se comparó el número de individuos por hectárea en seis bosques tropicales de América, Asia y África, se evidenció que en todos los casos hubo una disminución importante conforme se restringía la evaluación a clases de diámetro mayores; de este modo, considerando árboles con DAP igual o mayor a $30 \mathrm{~cm}$, sólo se registraron entre el 12 y $19 \%$ respecto del total registrado a partir de $10 \mathrm{~cm}$ (LaFrankie et al., 2006).

Los valores hallados sugieren que una evaluación de solo árboles de diámetros mayores a $40 \mathrm{~cm}$, tendría una resolución muy baja del componente de Diversidad Alfa arbórea en el espacio estudiado. La Diversidad Alfa, medida con el índice Alfa de Fisher (por ha) evidencia una importante disminución en las clases diamétricas mayores (en promedio se reduce de 46.9 a 28.4 para los bosques premontanos y de 34.4 a 19.6 para los bosques montanos). Esta tendencia a la diminución de la captura de la diversidad ta mbién se ha registrado en los bosques premontanos evaluados a partir de unidades de muestreo que incluyen diámetros a partir de $2.5 \mathrm{~cm}$ (Transectos RAP), en donde hay una clara reducción tanto de la abundancia como de la riqueza de especies. Un estudio, que incluyó todos los árboles con diámetros $\geq 1 \mathrm{~cm}$ de DAP, mostró que el valor de índice fue casi el mismo (39.4/ha) que cuando solo se consideraron los árboles con un diámetro $<10$ cm de DAP (40.1); mientras que, para árboles de diámetro $\geq 10 \mathrm{~cm}$ de DAP el valor del índice fue mucho menor (30.8/ha) (Memiaghe et al., 2016). Si bien evaluaciones que incluyan diámetros por debajo de los $10 \mathrm{~cm}$ y más de $2.5 \mathrm{~cm}$ de DAP requieren una mayor inversión de recursos, es importante valorar la pérdida de información que podría estarocurriendo.

A nivel regional, las familias Melastomataceae, Piperaceae y Malvaceae fueron registradas principalmente en las clases de diamétricas inferiores en bosques tropicales americanos (LaFrankie et al., 2006). Adicionalmente, recientes estudios sobre la flora leñosa (a partir de $2.5 \mathrm{~cm}$ de DAP), en nuestro mismo ámbito de estudio, muestran que Piperaceae es un

Tabla 13. Número (y porcentaje) de familias en función del diámetro mínimo de evaluación en los Transectos RAP evaluados (estrato premontano) de la Selva Centraldel Perú.

\begin{tabular}{|c|c|c|c|c|c|}
\hline \multirow{2}{*}{$\begin{array}{l}\text { Código de } \\
\text { Transecto }\end{array}$} & \multicolumn{5}{|c|}{ Número total de familias APG (Porcentaje) } \\
\hline & $>2.5 \mathrm{~cm} \mathrm{DAP}$ & $>10 \mathrm{~cm} \mathrm{DAP}$ & $>20 \mathrm{~cm} \mathrm{DAP}$ & $>30 \mathrm{~cm} \mathrm{DAP}$ & $>40 \mathrm{~cm} \mathrm{DAP}$ \\
\hline $\mathrm{T} 1-30$ & $21(100)$ & $12(57.1)$ & $9(42.9)$ & $8(38.1)$ & $6(28.6)$ \\
\hline $\mathrm{T} 2-30$ & $21(100)$ & $13(61.9)$ & $9(42.9)$ & $4(19)$ & $4(19)$ \\
\hline $\mathrm{T} 1-40$ & $23(100)$ & $15(65.2)$ & $12(52.2)$ & $9(39.1)$ & $4(17.4)$ \\
\hline $\mathrm{T} 2-40$ & $22(100)$ & $13(59.1)$ & $10(45.5)$ & $8(36.4)$ & $6(27.3)$ \\
\hline T3-40 & $26(100)$ & $20(76.9)$ & $11(42.3)$ & $3(11.5)$ & $2(7.7)$ \\
\hline $\mathrm{T} 1>50$ & $25(100)$ & $14(56)$ & $9(36)$ & $9(36)$ & $3(12)$ \\
\hline $\mathrm{T} 2>50$ & $31(100)$ & $22(71)$ & $13(41.9)$ & $8(25.8)$ & $5(16.1)$ \\
\hline $\mathrm{T} 3>50$ & $27(100)$ & $19(70.4)$ & $10(37)$ & $7(25.9)$ & $3(11.1)$ \\
\hline
\end{tabular}


componente principalde bosques jóvenes (en las clases diamétricas menores) y que Moraceae es un componente principal de bosques maduros (en las clases diamétricas mayores) (Echía \& Reynel, 2019; Quintero et al., 2020). Puede haber importantes diferencias a nivel local, más aun considerando el ensamblaje de especies en ecosistemas montañosos como el estudiado. Los cambios en la composición florística, conforme nos acercamos a las clases diamétricas superiores, podrían ser sugerentes si se analizan desde una perspectiva funcional. Una estructura de alto número de individuos en las clases diamétricas menores ayuda a garantizar el establecimiento futuro de los árboles que ocuparán el dosel del bosque. Además, se ha sugerido que los bosques tropicales, a pesar de presentar similar número de individuos, áreas basal y taxonomía de árboles, ocupan la estructura del dosel de forma diferente (LaFrankie et al., 2006). Por otro lado, la reducción del número de individuos registrados en las evaluaciones reduce las posibilidades de incorporar especies raras o representadas por un solo individuo.

Dado que, los altos niveles de diversidad dentro de las clases de menor diámetro pueden dar a estos bosques altos niveles de resistencia estructural a las perturbaciones antropogénicas / naturales y a un clima cambiante (Memiaghe et al., 2016), es importante considerar el análisis de la diversidad de especies en rangos de diámetros más a mplios, como los mostrados en este estudio. Considerando que los bajos niveles de riqueza de especies de árboles de diámetros mayores (> $60 \mathrm{~cm}$ de DAP) ha sido asociada a especies comúnmente conformadas por pocos individuos (Lutz et al., 2018), la estructura de las clases diamétricas menores requiere ser incluido en el análisis de la regeneración del dosel superior.

Finalmente, los árboles de diámetros mayores (> 60 $\mathrm{cm}$ de DAP) contribuyen con aproxima damente el $41 \%$ de la biomasa forestal, por lo que es importante considerar su dinámica y sensibilidad al cambio a mbiental (Lutz et al., 2018), y para ello es necesario incluir en las evaluaciones las clases de diámetro menores, donde se registraría la regeneración natural de esas especies importantes. No obstante, son muy escasas las publicaciones que documentan este importante aspecto. Más aún, los esfuerzos por monitorear los bosques considerando individuos con diámetros menores a $10 \mathrm{~cm}$ son considerablemente menores, no sólo en el Neotrópico (Memiaghe et al., 2016), lo que limita la comprensión de la estructura, dinámica y diversidad de árboles de los bosques tropicales, considerando la regeneración de los árboles que ocupan el dosel superior y los árboles que nunca alcanzan los $10 \mathrm{~cm}$ de DAP.

\section{Conclusiones}

Las evaluaciones de la diversidad arbórea considerando solamente diámetros a la altura del pecho
(DAP) $\geq 40 \mathrm{~cm}$ están bastante lejos de capturar el contenido de dicha diversidad en las áreas de bosque húmedo premontano y montano en la Selva Central del Perú. Considerando solamente diámetros a la altura del pecho $\geq 40 \mathrm{~cm}$, la diversidad arbórea promedio hallada equivale al $29.2 \%$ del a diversidad total en el caso de bosques húmedos premontanos ( 940 - $1150 \mathrm{msnm}), \mathrm{y}$ de $22.9 \%$ para el caso de bosques húmedos montanos (2078 - $2770 \mathrm{msnm}$ )

\section{Agradecimientos}

Los a utores a gra decen a todo el personaldelCírculo de Investigación DINAFOR de la Universidad Nacional Agraria La Molina (Perú) por su colaboración durante las fases de campo y gabinete. Esta investigación fue financiada por Cienciactiva del CONCYTEC (Perú).

\section{Literatura citada}

Antón D. \& Reynel C. (Eds). 2004. Relictos de bosque de excepcional diversidad en los Andes Centrales de Perú. Herbario de la Facultad de Ciencias Forestales / Universidad Nacional Agraria La Molina y APRODES (Asociación Peruana para la Promoción del Desarrollo Sostenible). Darwin Initiative Project 09/017 y PBR (Proyecto de Investigación en Bosques Ribereños UNALM/FIU).

http://cdc.lamolina.edu.pe/treediversity/Relictos\%20de $\% 20$ bosques 1.pdf.

APG (The Angiosperm Phylogeny Group). 2016. An update of the Angiosperm Phylogeny Group classification for the orders and families of flowering plants: APG IV. Botanical Journal of the Linnean Society, 181(1): 1-20. DOI: https://doi.org/10.1111/boj.12385.

Bordenave B.G., de Granville J.-J. \& Hofs M. 1998. Measurement of species richness of vascular plants in a neotropical rain forest in French Guiana. In: Dallmeier F. \& Comiskey J.A. (Eds.) Forest biodiversity in North, Central and South America, and the Caribbean. 411-425. Man and Biosphere Series, Chapter 21. Parthenon Press, UNESCO. https://horizon.documentation.ird.fr/exldoc/pleins_textes/pleins_textes_6/b_fdi_4950/010018241.pdf.

Bridson D. \& Forman L. 1999. The Herbarium Handbook. Royal Botanic Gardens, Kew.

Caro S., Reynel C. \& Antón D. 2004. Diversidad y composición de la flora arbórea en un área de ladera de colinas en bosque premontano: Fundo Génova-UNALM, Valle de Chanchamayo, 1000-1500 msnm. En: Antón D. \& Reynel C. (Eds.). Relictos de bosque de excepcional diversidad en los Andes centrales de Perú. 187220. Herbario de la Facultad de Ciencias Forestales / Universidad Nacional Agraria La Molina y APRODES (Asociación Peruana para la Promoción del Desarrollo Sostenible). Darwin Initiative Project 09/017 y PBR (Proyecto de Investigación en Bosques Ribereños UNALM/FIU).

http://cdc.lamolina.edu.pe/treediversity/Relictos\%20de $\% 20$ bosques1.pdf. 
Cava B., Coscarón M. \& Corronca J. 2015. Inventario y estimación específica de artrópodos en bosques del Noreste de Argentina. Revista Colombiana de Entomología, 41(1): 139-146. $\mathrm{http}: / /$ ref.scielo.org/jkykzy.

Colwell R.K. 2013. EstimateS: Statistical Estimation of Species Richness and Shared Species from Samples, 9.1.0 User's Guide.

http://viceroy.eeb.uconn.edu/EstimateS/EstimateSPages/ EstSUsersGuide/EstimateSUsersGuide.htm\#References.

De Rutte J. \& Reynel C. 2016. Composición y diversidad arbórea en la cumbre del bosque montano nublado Puyu Sacha, Chanchamyo, Dp. Junín, Perú. Centro de Estudios en Dendrología / Universidad Nacional Agraria La Molina.

http://cdc.lamolina.edu.pe/Descargas/CountDownload.p hp?y_Ruta=HerbarioMOL\&y_Archivo=ComposicionDi versidadArboreaPuyuSacha.

Dinerstein E., Joshi A.R., Vynne C., Lee A.T.L., PharandDeschênes F., França M., Fernando S., Birch T., Burkart K., Asner G.P. \& Olson D. 2020. A "Global Safety Net" to reverse biodiversity loss and stabilize Earth's climate. Science Advances, 6(36): eabb2824. DOI: https://doi.org/10.1126/sciadv.abb2824.

Echía E., Reynel C. \& Manta M. 2019. La flora leñosa establecida luego de las quemas en el valle de Chanchamayo - Selva central del Perú. Revista Forestal del Perú, 34(1): 83-101. DOI: http://dx.doi.org/10.21704/rfp.v34i1.1287.

Gentry A. 1988. Tree species richness of upper Amazonian forests. Proceedings of the National Academy of Sciences, 85(1): 156-159. DOI: https://doi.org/10.1073/pnas.85.1.156.

Gentry A. 1989. Diversidad florística y fitogeográfica de la Amazonía. En: Memorias del Simposio Internacional sobre Investigación y Manejo de la Amazonia. Instituto Nacional de los Recursos Naturales y el Medio Ambiente, Ministerio de Agricultura, Colombia.

Gentry A. 1995. Patterns of Diversity and Floristic Composition in Neotropical Montane Forests. 103-126 In: Churchill S., Balslev H., Forero E. \& Luteyn J. Biodiversity and Conservation of Neotropical Montane Forests: Proceedings Neotropical Montane Forest Biodiversity and Conservation Symposium (1993, Bronx, N.Y., USA). The New York Botanical Garden Press, New York.

Hammer Ø., Harper D.A.T. \& Ryan P. D. 2001. PAST: Paleontological Statistics Software Package for Education and Data Analysis. Paleontología Electrónica, 4(1): art.4: 1-9.

https://palaeo-electronica.org/2001_1/past/issue1_01.ht $\mathrm{m}$.

Holdridge L. 1978. Ecología basada en las zonas de vida. Centro Científico Tropical de Investigación y Enseñanza, CATIE, Turrialba, Costa Rica.

Honorio E. \& Reynel C. 2003. Vacíos en la colección de la flora de los Bosques Húmedos del Perú. Herbario de la Facultad de Ciencias Forestales, Universidad Nacional Agraria La Molina.

INEI (Instituto Nacional de Estadística e Informática). 1993. Censo IX de Población y IV de Vivienda - Estadísticas de Centros Poblados. Instituto Nacional de Estadística e Informática, Lima
INRENA (Instituto Nacional de Recursos Naturales). 1995. Mapa ecológico del Perú. Mapa y Guía explicativa (Actualización del mismo elaborado por ONERN, 1976). Instituto Nacional de Recursos Naturales, Lima.

Joppa L., Roberts L., Myers N. \& Pimme S. 2011. Biodiversity hotspots house most undiscovered plant species. Proceedings of the National Academy of Science, 108(32): 13171-13176.

DOI: https://doi.org/10.1073/pnas.1109389108.

LaFrankie J.V., Ashton P.S., Chuyong G.B., Co L., Condit R., Davies S.J., Foster R., Hubbell S.P., Kenfack D., Lagunad D., Losos E.C., Nor N.S., Tan S., Thomas D.W., Valencia R. \& Villa G. 2006. Contrasting structure and composition of the understory in species-rich tropical rain forests. Ecology, 87(9): 2298-2305. DOI: https://doi.org/10.1890/0012-9658(2006)87[2298: CSACOT]2.0.CO;2.

Lutz J., Furniss T., Johnson D., Davies S., Allen D., Alonso A., Anderson-Teixeira K., Andrade A., Baltzer J., Becker K., Blomdahl E., Bourg N., Bunyavejchewin S., Burslem D., Cansler A., Cao K., Cao M., Cardenas D., Chang L., Chao K., Chao W., Chiang J., Chu C., Chuyong G., Clay K., Condit R., Cordell S., Dattaraja H., Duque A., Ewango C., Fischer G., Fletcher C., Freund J., Giardina C., Germain S., Gilbert G., Hao Z., Hart T., Hau B., He F., Hector A., Howe R., Hsieh C., Hu Y., Hubbell S., Inman-Narahari F., Itoh A., Janík D., Kassim A., Kenfack D., Korte L., Kral K., Larson A., Li Y., Lin Y., Liu S., Lum S., Ma K., Makana J., Malhi Y., McMahon S., McShea W., Memiaghe H., Mi X., Morecroft M., Musili P., Myers J., Novotny V., de Oliveira A., Ong P., Orwig D., Ostertag R., Parker G., Patankar R., Phillips R., Reynolds G., Sack L., Song G., Su S., Sukumar R., Sun I., Suresh H., Swanson M., Tan S., Thomas D., Thompson J., Uriarte M., Valencia R., Vicentini A., Vrska T., Wang X., Weiblen G., Wolf A., Wu S., Xu S., Yamakura T., Yap S. \& Zimmerman J. 2018. Global importance of large-diameter trees. Global Ecology and Biogeography 27(7): 849-864. DOI: https://doi.org/10.1111/geb.12747.

Maden K. 2004. Plant Collection and Herbarium Techniques. Our Nature, 2(1): 53-57. DOI: https://doi.org/10.3126/on.v2i1.327.

Malleux M.O. 1982. Inventarios forestales en bosques tropicales. Universidad Nacional Agraria La Molina. Lima-Perú.

Marcelo J. \& Reynel C. 2014. Patrones de diversidad y composición florística de parcelas de evaluación permanente en la selva central del Perú. Rodriguesia, 65(1): 35-47.

DOI: $10.1590 / \mathrm{S} 217578602014000100003$.

Memiaghe H.R., Lutz J.A., Korte L., Alonso A. \& Kenfack D. 2016. Ecological Importance of Small-Diameter Trees to the Structure, Diversity and Biomass of a Tropical Evergreen Forest at Rabi, Gabon. PLOS ONE, 11(5): e0154988. DOI: https://doi.org/10.1371/journal.pone.0154988.

MINAG. 2012. Manual base para la Planificación y Ejecución de Inventarios Forestales en Bosques de Producción Permanente. Versión 1.0. Ministerio de Agricultura (MINAG). s.e. Lima / Perú. https://www.midagri.gob.pe/portal/download/pdf/marcol egal/normaslegales/resolucionesministeriales/2012/mayo /manual_inv_forestales.pdf. 
Mwavu E. \& Witkowski E. 2015. Woody species alphadiversity and species abundance in an African semideciduous tropical rain forest. Biotropica, 47(4): 424-434. DOI: https://doi.org/10.1111/btp.12223.

Phillips O. \& Miller J. 2002. Global patterns of plant diversity: Alwyn H. Gentry's forest transect data set. Monographs in Systematic Botany from the Missouri Botanical Garden. St. Louis, Missouri. U.S.A.

Phillips O., Vásquez R., Núñez P., Monteagudo A., Chuspe M., Galiano W., Peña A., Timaná M., Yli-Halla M. \& Rose S. 2003. Efficient plot-based floristic assessment of tropical forests. J. Trop. Ecology, 19(6): 629-645. DOI: https://doi.org/10.1017/S0266467403006035.

Phillips O., Baker T., Feldpausch T. \& Brienen R. 2009. RAINFOR Manual de campo para la remedición y establecimiento de parcelas permanentes. http://www.rainfor.org/upload/ManualsSpanish/RAINF OR\%20manual\%20de\%20campo\%20version\%20Junio $\% 202009 \% 20$ ESP.pdf.

Pitman N., Terborgh J., Silman M., Nuñez P., Neill D., Cerón C., Palacios W. \& Aulestia M. 2002. A comparison of tree species diversity in two upper Amazonian forests. Ecology, 83(11): 3210-3224.

DOI: https://doi.org/10.1890/0012-9658(2002)083[3210 :ACOTSD]2.0.CO;2.

Quintero F., Cáceres B., Reynel C., Fernandez-Hilario R., Wong A., Chávez J. \& Palacios S. 2020. Tiempos de recomposición de la diversidad arbórea a lo lago de la sucesión vegetal en los bosques del valle de Chanchamayo / Junín / Perú. Ecología Aplicada, 19(2): 111-120.

DOI: http://dx.doi.org/10.21704/rea.v19i2.1562.

RAINFOR. 2000. Red Amazónica de Inventarios Forestales. Consultado el 15 de mayo de 2019 de: http://www.rainfor.org/es.
Reynel C. \& Honorio E. 2004. Diversidad y composición de la flora arbórea en un área de ladera de bosque montano: Pichita, valle de Chanchamayo 2000-2500 msnm. En: Antón D. \& Reynel C. (Eds.). Relictos de bosque de excepcional diversidad en los Andes centrales de Perú. 45-98. Herbario de la Facultad de Ciencias Forestales / Universidad Nacional Agraria La Molina y APRODES (Asociación Peruana para la Promoción del Desarrollo Sostenible). Darwin Initiative Project 09/017 y PBR (Proyecto de Investigación en Bosques Ribereños UNALM/FIU).

http://cdc.lamolina.edu.pe/treediversity/Relictos\%20de $\% 20$ bosques 1.pdf.

Ter Steege H., Pitman N., Sabatier D., Castellanos H., Van der Hout P., Daly D., Silveira M., Phillips O., Vasquez R., Van Andel T., Duivenvoorden J., Adalardo A., Ek R., Lilwah R., Thomas R., Van Essen J., Baider C., Maas J., Mori S., Terborgh J., Nuñez P., Mogollon H. \& Morawetz H. 2003. A spatial model of tree a-diversity and tree density for the amazon. Biodiversity and Conservation, 12(11): 2255-2277.

DOI: https://doi.org/10.1023/A:1024593414624.

Vásquez R. \& Gentry A. 1987. Limitaciones del uso de nombres vernaculares en los inventarios forestales de la Amazonia. Revista Forestal del Perú, 14(1): 109-120. https://revistas.lamolina.edu.pe/index.php/rfp/article/vie $\mathrm{w} / 144$.

Villareal H., Álvarez M., Córdoba S., Escobar F., Fagua G., Gast F., Mendoza H., Ospina M. \& Umaña A. 2004. Manual de métodos para el desarrollo de inventarios de biodiversidad. Programa de Inventarios de Biodiversidad. Instituto de Investigación de Recursos Biológicos Alexander von Humboldt. Segunda edición. Bogotá, Colombia.

URI: http://hdl.handle.net/20.500.11761/31419. 


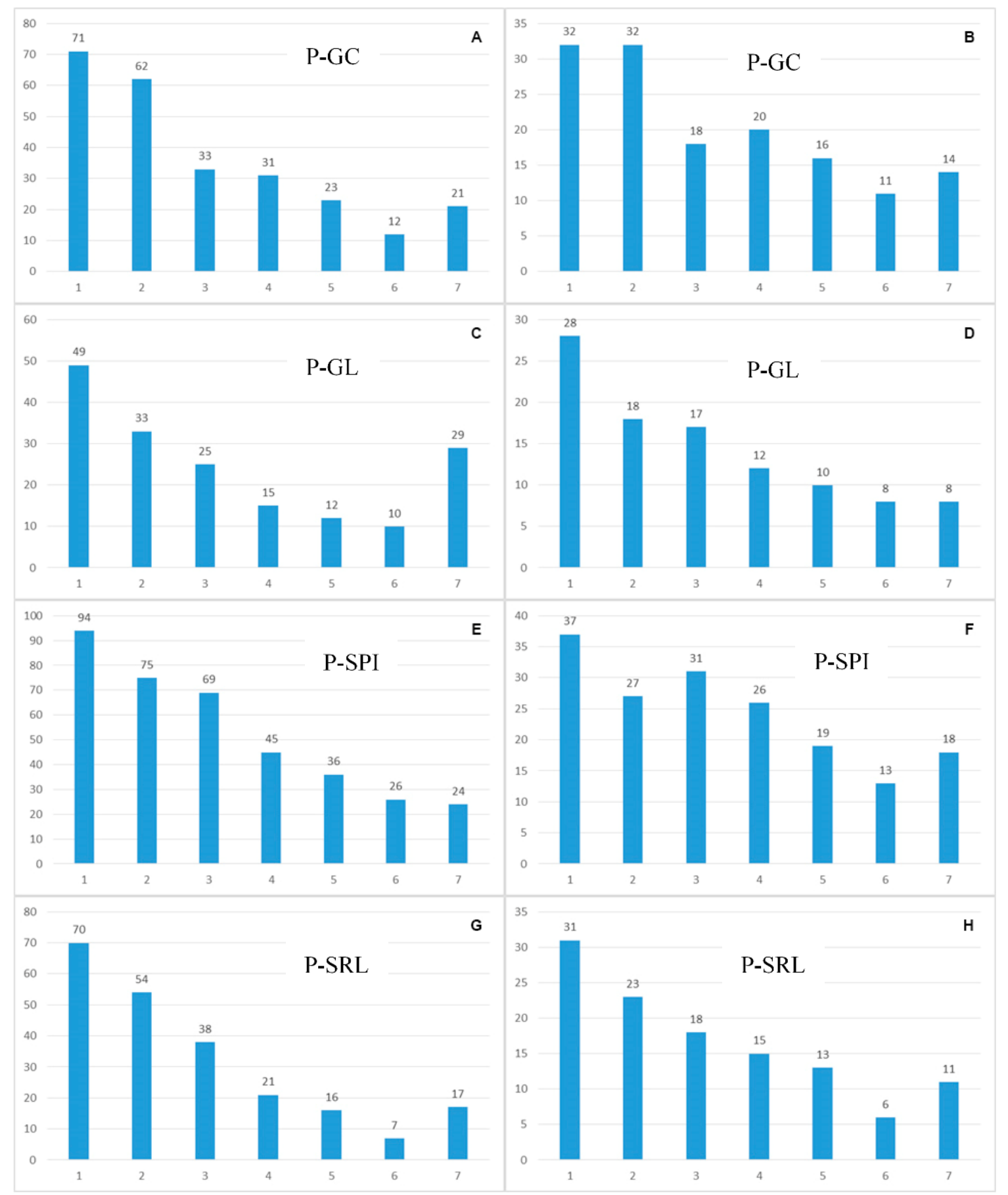

Leyenda: A, C, E y G: número de especies. B, D, F, y H: número de familias.

Figura 2. Número de especies y familias en función de las cla ses dia métricas, para las Parcelas Permanentes ubicadas en el estrato premontano. 


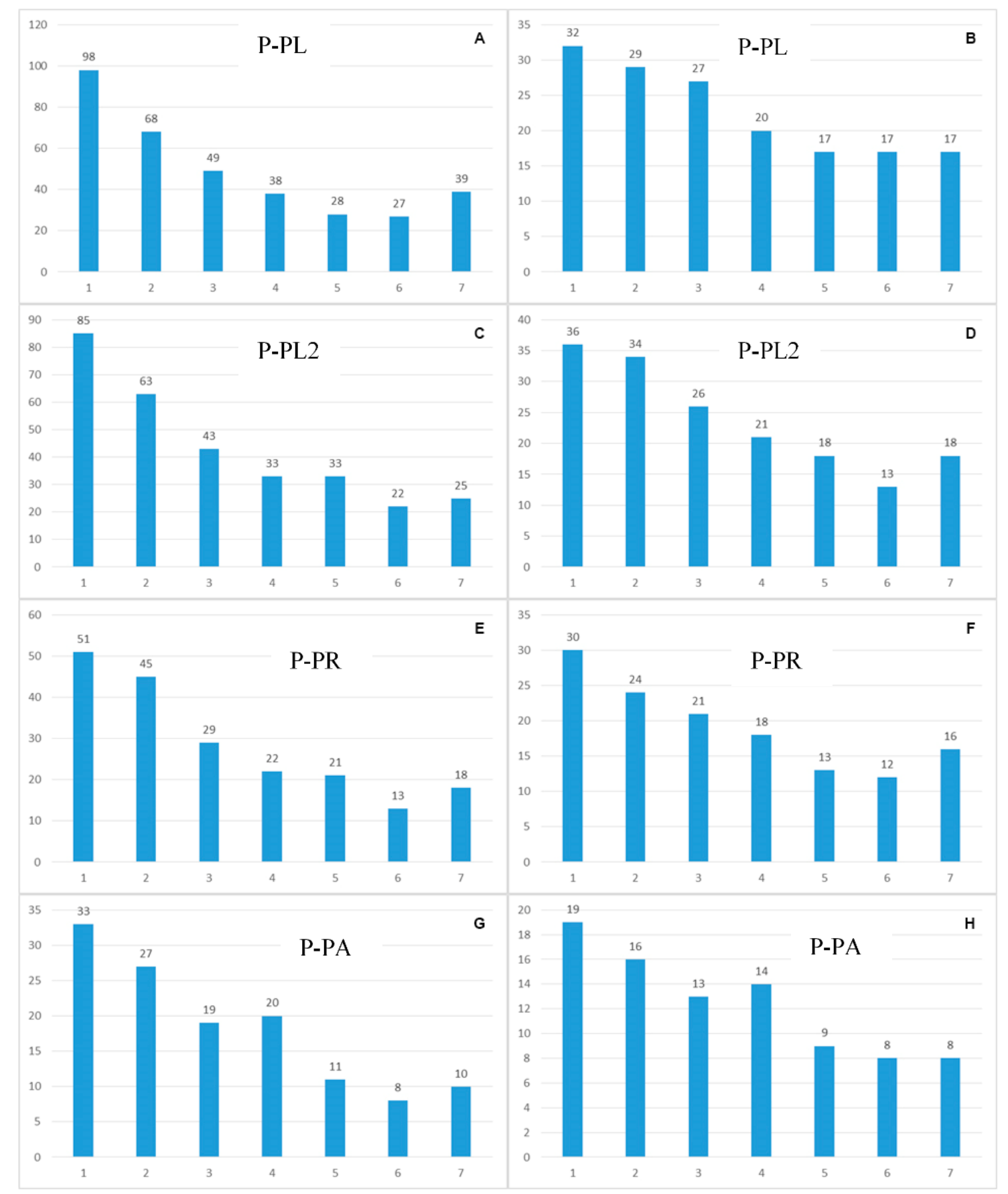

Leyenda: A, C, E y G: número de especies. B, D, F, y H: número de familias.

Figura 3. Número de especies y familias en función de las clases diamétricas, para las Parcelas Permanentes ubicadas en el estrato montano. 


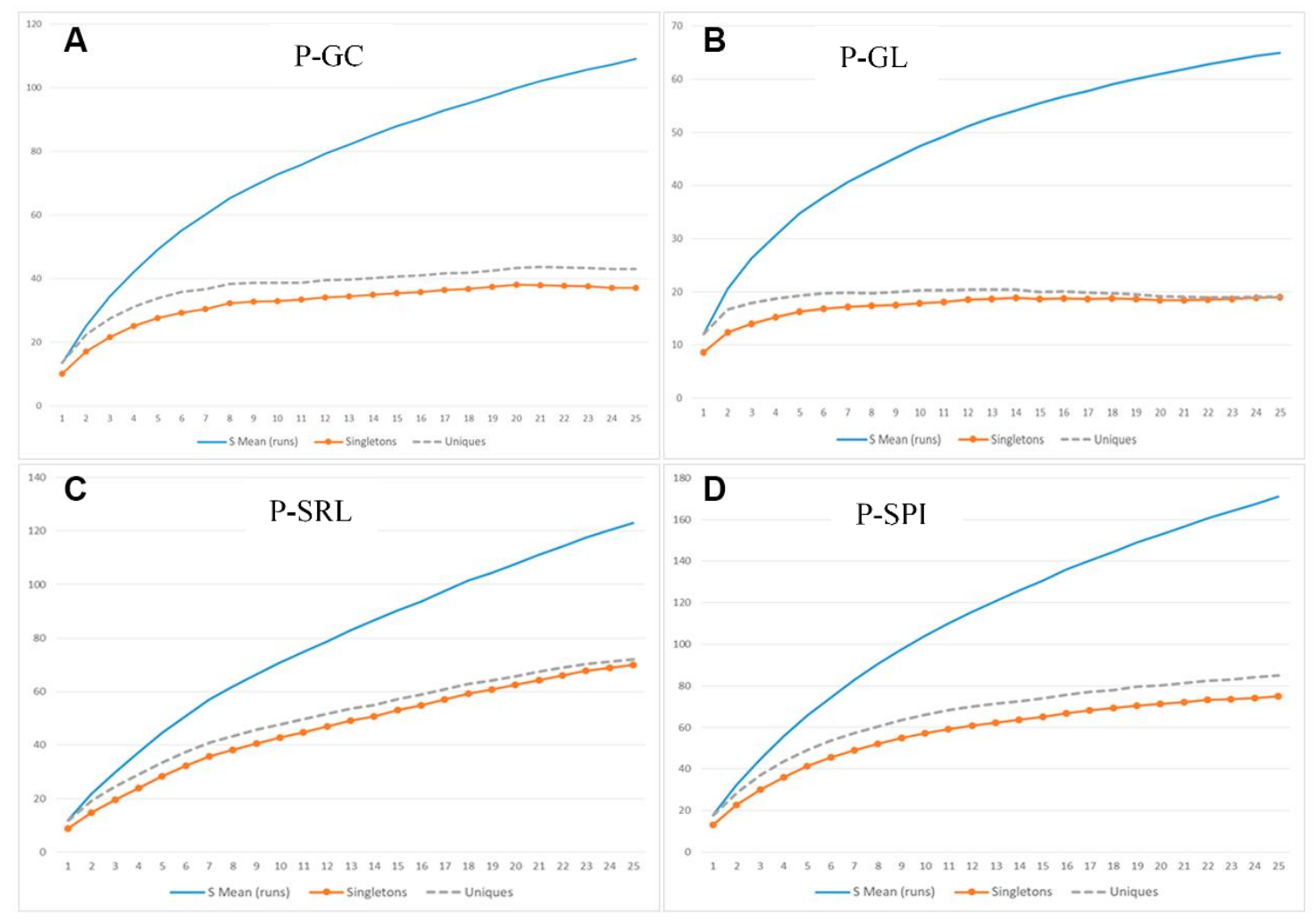

Figura 4. Curvas de acumulación de especies para las Parcelas Permanentes ubicadas en el estrato premontano.
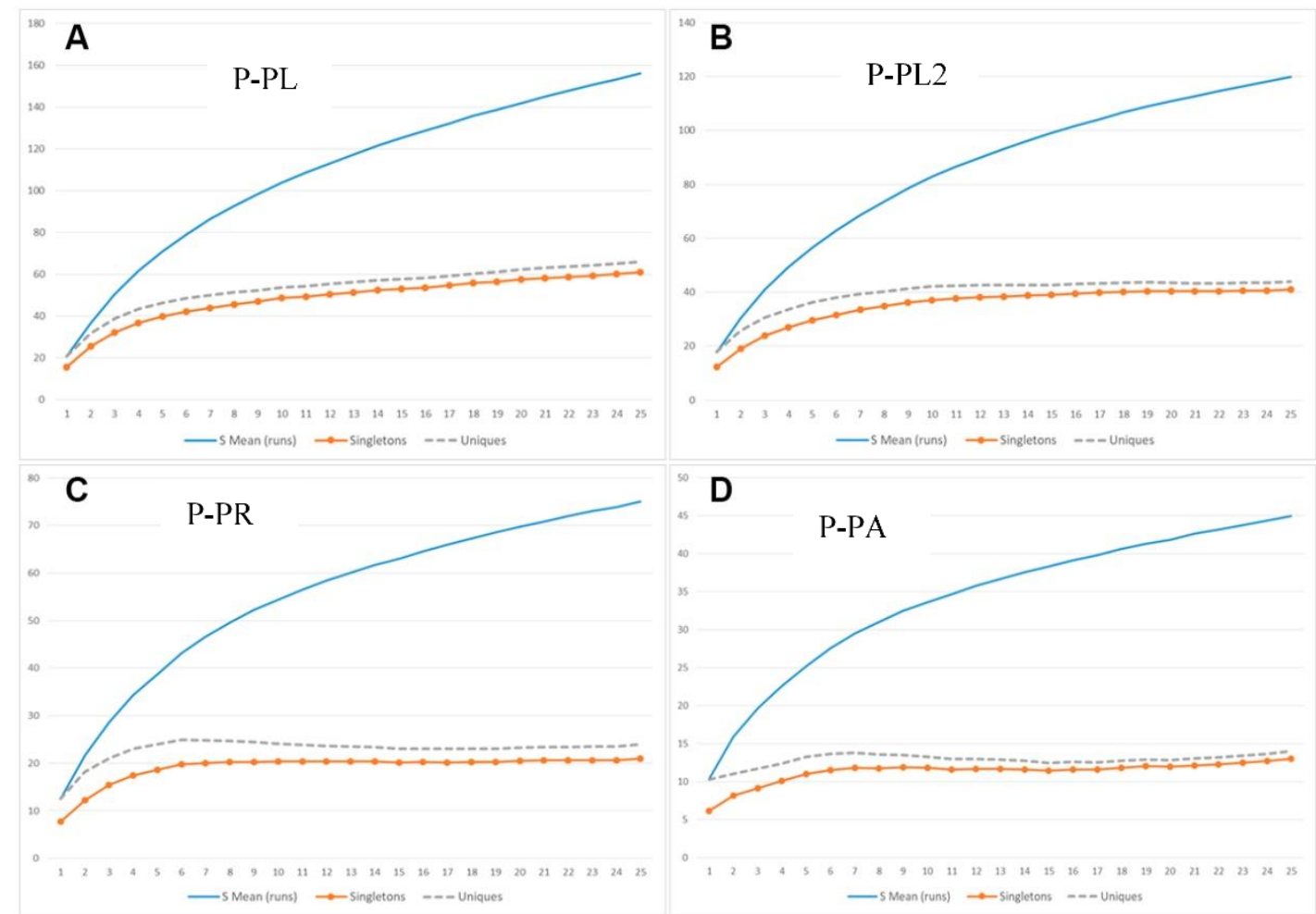

Figura 5. Curvas de acumulación de especies para las Parcelas Permanentes ubicadas en el estrato montano. 
Tabla 8. Géneros con individuos con un diámetro (DAP) mayor a $40 \mathrm{~cm}$ en las Parcelas Permanentes evaluadas en el estra to premontano de la Selva Central del Perú.

\begin{tabular}{|c|c|c|c|}
\hline P-GC & P-GL & P-SRL & P-SPI \\
\hline $\begin{array}{lr}\text { Alchornea, Aspidosperma, } \\
\text { Batocarpus, } & \text { Cariniana, } \\
\text { Celtis, } & \text { Clarisia, } \\
\text { Dendropanax, } & \\
\text { Endlicheria, } & \text { Huertea, } \\
\text { Inga, } & \text { Licaria, } \\
\text { Margaritaria, Mauria, } & \text { Meliosma, Myrcianthes, } \\
\text { Nectandra, } & \text { Ocotea, } \\
\text { Pseudolmedia, Terminalia }\end{array}$ & 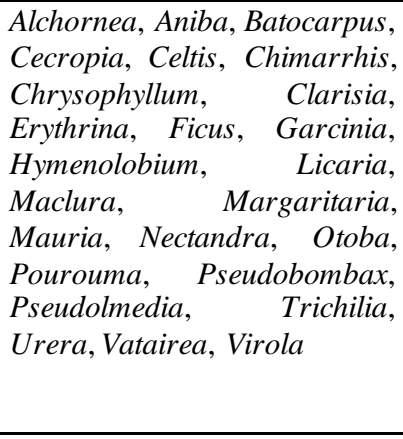 & $\begin{array}{l}\text { Alchornea, Bixa, Cecropia, } \\
\text { Ceiba, Cosmibuena, Ficus, } \\
\text { Ladenbergia, Macbrideina, } \\
\text { Machaerium, Nectandra, } \\
\text { Ochroma, } \quad \text { Pourouma, } \\
\text { Sciodaphyllum, Tachigali, } \\
\text { Trichilia, Trophis, Virola }\end{array}$ & $\begin{array}{lr}\text { Alchornea, } & \text { Cecropia, } \\
\text { Clarisia, } & \text { Dendropanax, } \\
\text { Drypetes, } & \text { Endlicheria, } \\
\text { Eschweilera, } & \text { Eugenia, } \\
\text { Geonoma, } & \text { Miconia, } \\
\text { Myrsine, } & \text { Oenocarpus, } \\
\text { Oreopanax, } & \text { Ormosia, } \\
\text { Pleuranthodendron, } \\
\text { Pourouma, } \\
\text { Sciodaphyllum, Senefeldera, } \\
\text { Simira, } \\
\text { Trattinnickia, Virola } \\
\end{array}$ \\
\hline
\end{tabular}

Tabla 9. Géneros con individuos de un diámetro (DAP) mayor a $40 \mathrm{~cm}$ en las Parcelas Permanentes evaluadas en el estrato montano de la Selva Centraldel Perú.

\begin{tabular}{|c|c|c|c|}
\hline P-PL & P-PL2 & P-PR & P-PA \\
\hline $\begin{array}{lr}\text { Alchornea, } & \text { Alchorneopsis, } \\
\text { Aniba, } & \text { Beilschmiedia, } \\
\text { Cecropia, } & \text { Coussapoa, } \\
\text { Cyathea, } & \text { Elaeagia, } \\
\text { Faramea, Ficus, Guatteria, } & \text { Geliocarpus, } \\
\text { Huertea, } & \text { Mieronyma, } \\
\text { Nectandra, Ocotea, Piper, } & \text { Pagnolia, } \\
\text { Podocarpus, } & \text { Protium, } \\
\text { Prumnopitys, } & \\
\text { Pseudolmedia, } & \text { Sapium, } \\
\text { Simira, } & \text { Tovomita, } \\
\text { Weinmannia } & \\
\end{array}$ & $\begin{array}{l}\text { Ampelocera, Aniba, } \\
\text { Coussapoa, Eugenia, Ficus, } \\
\text { Gordonia, } \\
\text { Huertea, Hieronyma, } \\
\text { Juglans, Magnolia, Morus, } \\
\text { Myrcia, } \\
\text { Nectandra, Persea, Piper, } \\
\text { Protium, Myrcianthes, } \\
\text { Prunus, Styrax, Symplocos, } \\
\text { Trema }\end{array}$ & 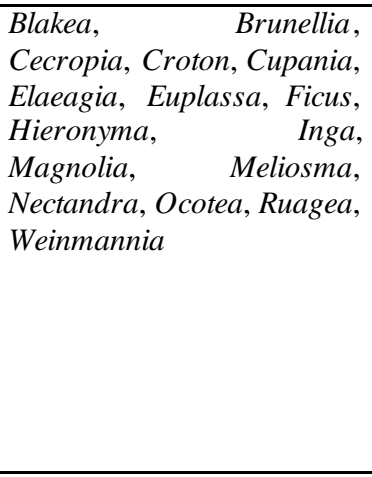 & $\begin{array}{l}\text { Clusia, Gordonia, Miconia, } \\
\text { Myrsine, } \\
\text { Podocarpus, Symplocos, } \\
\text { Ternstroemia, Weinmannia }\end{array}$ \\
\hline
\end{tabular}




\begin{tabular}{|c|c|c|c|c|c|c|c|c|c|c|c|c|c|c|c|c|}
\hline \multirow{2}{*}{$\begin{array}{l}\text { Clase } \\
\text { Diam }\end{array}$} & \multicolumn{2}{|c|}{ T1-30 } & \multicolumn{2}{|c|}{ T2-30 } & \multicolumn{2}{|c|}{ T1-40 } & \multicolumn{2}{|c|}{ T2-40 } & \multicolumn{2}{|c|}{ T3-40 } & \multicolumn{2}{|c|}{ T1>50 } & \multicolumn{2}{|c|}{ T2>50 } & \multicolumn{2}{|c|}{ T3>50 } \\
\hline & $\begin{array}{l}\text { Núm. } \\
\text { inds. } \\
(\%)\end{array}$ & $\begin{array}{l}\text { Núm. } \\
\text { spp. } \\
(\%)\end{array}$ & $\begin{array}{l}\text { Núm. } \\
\text { inds. } \\
(\%)\end{array}$ & $\begin{array}{l}\text { Núm. } \\
\text { spp. } \\
(\%)\end{array}$ & $\begin{array}{l}\text { Núm. } \\
\text { inds. } \\
(\%)\end{array}$ & $\begin{array}{l}\text { Núm. } \\
\text { spp. } \\
(\%)\end{array}$ & $\begin{array}{l}\text { Núm. } \\
\text { inds. } \\
(\%)\end{array}$ & $\begin{array}{c}\text { Núm. } \\
\text { spp. } \\
(\%)\end{array}$ & $\begin{array}{l}\text { Núm. } \\
\text { inds. } \\
(\%)\end{array}$ & $\begin{array}{l}\text { Núm. } \\
\text { spp. } \\
(\%)\end{array}$ & $\begin{array}{l}\text { Núm. } \\
\text { inds. } \\
(\%)\end{array}$ & $\begin{array}{c}\text { Núm. } \\
\text { spp. } \\
(\%)\end{array}$ & $\begin{array}{c}\text { Núm. } \\
\text { inds. } \\
(\%)\end{array}$ & $\begin{array}{l}\text { Núm. } \\
\text { spp. } \\
(\%)\end{array}$ & $\begin{array}{l}\text { Núm. } \\
\text { inds. } \\
(\%)\end{array}$ & $\begin{array}{l}\text { Núm. } \\
\text { spp. } \\
(\%)\end{array}$ \\
\hline 1 & 222 & 35 & 253 & 38 & 346 & 58 & 229 & 42 & 330 & 45 & 339 & 46 & 282 & 45 & 285 & 46 \\
\hline & (36.9) & $(62.5)$ & $(42.2)$ & $(69.1)$ & $(50.7)$ & $(72.5)$ & (39.6) & (66.7) & (51.9) & (80.4) & $(47.8)$ & (73) & $(41.2)$ & (60) & $(46.8)$ & $(75.4)$ \\
\hline 2 & $\begin{array}{c}228 \\
(37.9)\end{array}$ & $\begin{array}{c}41 \\
(73.2)\end{array}$ & $\begin{array}{l}186 \\
(31)\end{array}$ & $\begin{array}{l}33 \\
(60)\end{array}$ & $\begin{array}{c}195 \\
(28.6)\end{array}$ & $\begin{array}{l}48 \\
(60)\end{array}$ & $\begin{array}{c}160 \\
(27.7)\end{array}$ & $\begin{array}{c}36 \\
(57.1)\end{array}$ & $\begin{array}{c}163 \\
(25.6)\end{array}$ & $\begin{array}{c}37 \\
(66.1)\end{array}$ & $\begin{array}{c}176 \\
(24.8)\end{array}$ & $\begin{array}{c}35 \\
(55.6)\end{array}$ & $\begin{array}{c}187 \\
(27.3)\end{array}$ & $\begin{array}{c}34 \\
(45.3)\end{array}$ & $\begin{array}{l}134 \\
(22)\end{array}$ & $\begin{array}{c}37 \\
(60.7)\end{array}$ \\
\hline 3 & $\begin{array}{c}62 \\
(10.3)\end{array}$ & $\begin{array}{c}24 \\
(42.9)\end{array}$ & $\begin{array}{c}67 \\
(11.2)\end{array}$ & $\begin{array}{c}26 \\
(47.3)\end{array}$ & $\begin{array}{c}55 \\
(8.1)\end{array}$ & $\begin{array}{c}22 \\
(27.5)\end{array}$ & $\begin{array}{c}61 \\
(10.6)\end{array}$ & $\begin{array}{c}24 \\
(38.1)\end{array}$ & $\begin{array}{c}66 \\
(10.4)\end{array}$ & $\begin{array}{c}21 \\
(37.5)\end{array}$ & $\begin{array}{c}68 \\
(9.6)\end{array}$ & $\begin{array}{c}23 \\
(36.5)\end{array}$ & $\begin{array}{c}96 \\
(14)\end{array}$ & $\begin{array}{c}17 \\
(22.7)\end{array}$ & $\begin{array}{c}88 \\
(14.4)\end{array}$ & $\begin{array}{c}24 \\
(39.3)\end{array}$ \\
\hline 4 & $\begin{array}{c}34 \\
(5.7)\end{array}$ & $\begin{array}{c}16 \\
(28.6)\end{array}$ & $\begin{array}{c}29 \\
(4.8)\end{array}$ & $\begin{array}{c}15 \\
(27.3)\end{array}$ & $\begin{array}{c}28 \\
(4.1)\end{array}$ & $\begin{array}{c}18 \\
(22.5)\end{array}$ & $\begin{array}{c}43 \\
(7.4)\end{array}$ & $\begin{array}{c}16 \\
(25.4)\end{array}$ & $\begin{array}{c}23 \\
(3.6)\end{array}$ & $\begin{array}{c}14 \\
(25)\end{array}$ & $\begin{array}{l}57 \\
(8)\end{array}$ & $\begin{array}{c}23 \\
(36.5)\end{array}$ & $\begin{array}{c}47 \\
(6.9)\end{array}$ & $\begin{array}{c}20 \\
(26.7)\end{array}$ & $\begin{array}{c}47 \\
(7.7)\end{array}$ & $\begin{array}{c}26 \\
(42.6)\end{array}$ \\
\hline 5 & $\begin{array}{c}20 \\
(3.3)\end{array}$ & $\begin{array}{c}11 \\
(19.6)\end{array}$ & $\begin{array}{l}24 \\
(4)\end{array}$ & $\begin{array}{c}16 \\
(29.1)\end{array}$ & $\begin{array}{c}20 \\
(2.9)\end{array}$ & $\begin{array}{l}16 \\
(20)\end{array}$ & $\begin{array}{l}29 \\
(5)\end{array}$ & $\begin{array}{c}16 \\
(25.4)\end{array}$ & $\begin{array}{c}25 \\
(3.9)\end{array}$ & $\begin{array}{c}8 \\
(14.3)\end{array}$ & $\begin{array}{c}22 \\
(3.1)\end{array}$ & $\begin{array}{c}15 \\
(23.8)\end{array}$ & $\begin{array}{c}17 \\
(2.5)\end{array}$ & $\begin{array}{c}11 \\
(14.7)\end{array}$ & $\begin{array}{c}22 \\
(3.6)\end{array}$ & $\begin{array}{c}10 \\
(16.4)\end{array}$ \\
\hline 6 & $\begin{array}{c}13 \\
(2.2)\end{array}$ & $\begin{array}{c}9 \\
(16.1)\end{array}$ & $\begin{array}{c}17 \\
(2.8)\end{array}$ & $\begin{array}{c}8 \\
(14.5)\end{array}$ & $\begin{array}{l}13 \\
(1.9)\end{array}$ & $\begin{array}{c}9 \\
(11.3)\end{array}$ & $\begin{array}{c}19 \\
(3.3)\end{array}$ & $\begin{array}{c}9 \\
(14.3)\end{array}$ & $\begin{array}{l}19 \\
(3)\end{array}$ & $\begin{array}{c}5 \\
(8.9)\end{array}$ & $\begin{array}{l}20 \\
(2.8)\end{array}$ & $\begin{array}{c}14 \\
(22.2)\end{array}$ & $\begin{array}{l}15 \\
(2.2)\end{array}$ & $\begin{array}{c}12 \\
(16)\end{array}$ & $\begin{array}{l}12 \\
(2)\end{array}$ & $\begin{array}{c}10 \\
(16.4)\end{array}$ \\
\hline 7 & $\begin{array}{c}11 \\
(1.8)\end{array}$ & $\begin{array}{c}9 \\
(16.1)\end{array}$ & $\begin{array}{c}9 \\
(1.5)\end{array}$ & $\begin{array}{c}4 \\
(7.3)\end{array}$ & $\begin{array}{c}8 \\
(1.2)\end{array}$ & $\begin{array}{c}6 \\
(7.5)\end{array}$ & $\begin{array}{c}11 \\
(1.9)\end{array}$ & $\begin{array}{c}6 \\
(9.5)\end{array}$ & $\begin{array}{c}6 \\
(0.9)\end{array}$ & $\begin{array}{c}2 \\
(3.6)\end{array}$ & $\begin{array}{l}14 \\
(2)\end{array}$ & $\begin{array}{c}10 \\
(15.9)\end{array}$ & $\begin{array}{c}11 \\
(1.6)\end{array}$ & $\begin{array}{c}8 \\
(10.7)\end{array}$ & $\begin{array}{c}8 \\
(1.3)\end{array}$ & $\begin{array}{c}7 \\
(11.5)\end{array}$ \\
\hline 8 & $\begin{array}{c}1 \\
(0.2)\end{array}$ & $\begin{array}{c}1 \\
(1.8)\end{array}$ & $\begin{array}{c}2 \\
(0.3)\end{array}$ & $\begin{array}{c}2 \\
(3.6)\end{array}$ & $\begin{array}{c}4 \\
(0.6)\end{array}$ & $\begin{array}{c}3 \\
(3.8)\end{array}$ & $\begin{array}{c}7 \\
(1.2)\end{array}$ & $\begin{array}{c}6 \\
(9.5)\end{array}$ & $\begin{array}{c}2 \\
(0.3)\end{array}$ & $\begin{array}{c}1 \\
(1.8)\end{array}$ & $\begin{array}{c}8 \\
(1.1)\end{array}$ & $\begin{array}{c}7 \\
(11.1)\end{array}$ & $7(1)$ & $\begin{array}{c}5 \\
(6.7)\end{array}$ & $\begin{array}{c}5 \\
(0.8)\end{array}$ & $\begin{array}{c}5 \\
(8.2)\end{array}$ \\
\hline 9 & $\begin{array}{c}10 \\
(1.7) \\
\end{array}$ & $\begin{array}{c}7 \\
(12.5) \\
\end{array}$ & $\begin{array}{c}13 \\
(2.2) \\
\end{array}$ & $\begin{array}{c}5 \\
(9.1) \\
\end{array}$ & $\begin{array}{l}14 \\
(2)\end{array}$ & $\begin{array}{c}7 \\
(8.8) \\
\end{array}$ & $\begin{array}{c}19 \\
(3.3) \\
\end{array}$ & $\begin{array}{c}9 \\
(14.3) \\
\end{array}$ & $\begin{array}{c}2 \\
(0.3) \\
\end{array}$ & $\begin{array}{c}2 \\
(3.6) \\
\end{array}$ & $\begin{array}{c}5 \\
(0.7) \\
\end{array}$ & $\begin{array}{c}4 \\
(6.3) \\
\end{array}$ & $\begin{array}{c}22 \\
(3.2) \\
\end{array}$ & $\begin{array}{c}6 \\
(8) \\
\end{array}$ & $\begin{array}{c}8 \\
(1.3) \\
\end{array}$ & $\begin{array}{c}3 \\
(4.9) \\
\end{array}$ \\
\hline
\end{tabular}


NÚMERO DE ESPECIES POR DIÁMETRO MINNIMOEVALUADO EN SELVA CENTRAL DEL PERÚ

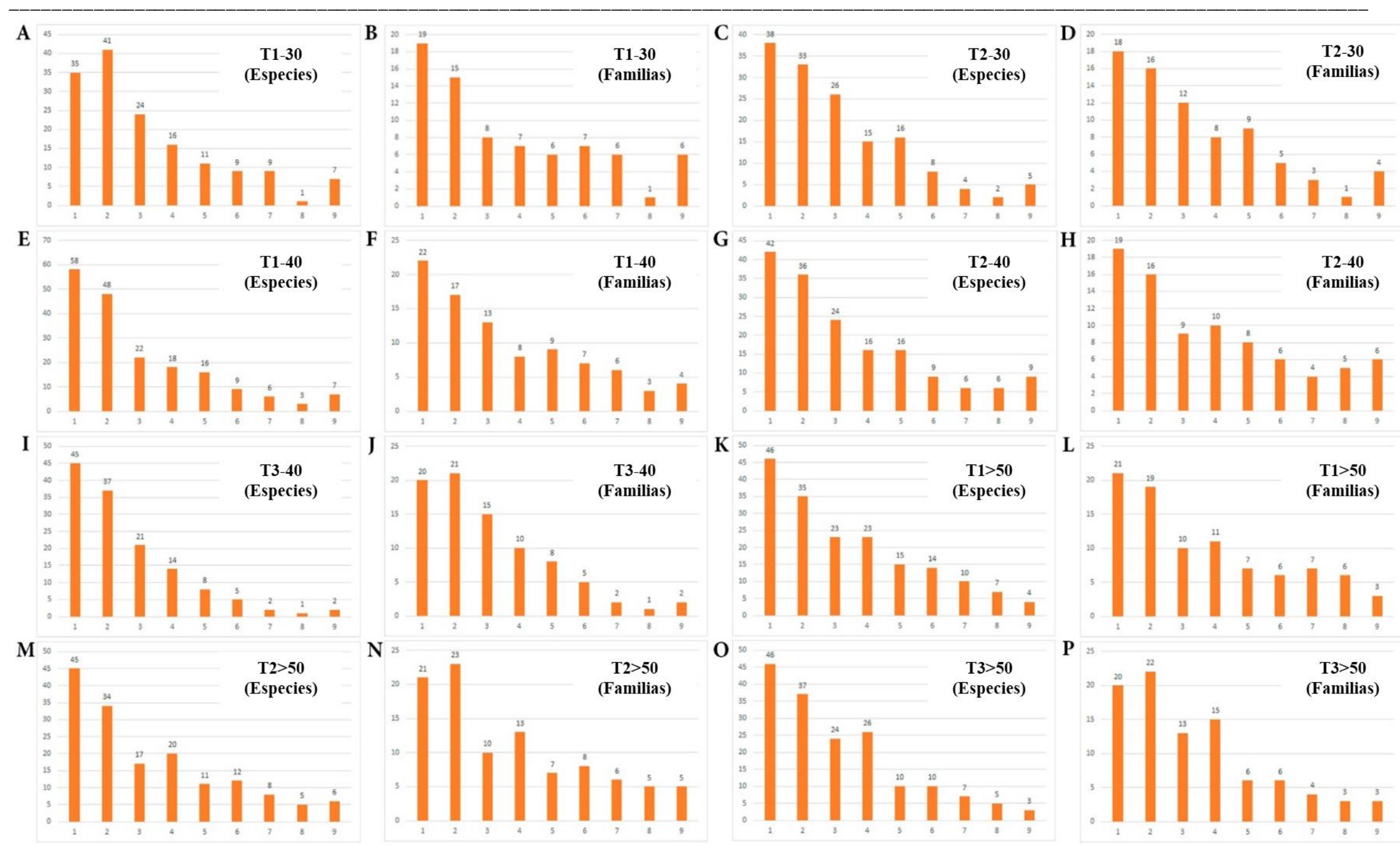

Figura 6. Número de especies y familias en función de las clases diamétricas, para los Transectos RAP ubicados en el estrato premontano. 
${ }^{1}$ Herbario Forestal MOL $\backslash$ Facultad de Ciencias Forestales, Universidad Nacional Agraria La Molina. Av. La Molina s/n / La Molina / Lima / Perú.

2 Autor de correspondencia: reynel@lamolina.edu.pe.

${ }^{3}$ Servicio Forestal y de Fauna Silvestre (SERFOR), Av. Javier Prado Oeste $N^{\circ} 2442$, Urb. Orrantia, Magdalena del Mar, Lima, Perú. 